\title{
Existence for Certain Systems of Nonlinear Fractional Differential Equations
}

\author{
Zhaowen Zheng, ${ }^{1}$ Xiujuan Zhang, ${ }^{1}$ and Jing Shao ${ }^{1,2}$ \\ ${ }^{1}$ School of Mathematical Sciences, Qufu Normal University, Qufu, Shandong 273165, China \\ ${ }^{2}$ Department of Mathematics, Jining University, Qufu, Shandong 273155, China \\ Correspondence should be addressed to Zhaowen Zheng; zhwzheng@126.com
}

Received 3 December 2013; Revised 22 February 2014; Accepted 17 March 2014; Published 6 April 2014

Academic Editor: Alberto Cabada

Copyright (C) 2014 Zhaowen Zheng et al. This is an open access article distributed under the Creative Commons Attribution License, which permits unrestricted use, distribution, and reproduction in any medium, provided the original work is properly cited.

By establishing a comparison result and using the monotone iterative technique, combining with the method of upper and lower solutions, the existence of solutions for systems of nonlinear fractional differential equations is considered. An example is given to demonstrate the applicability of our results.

\section{Introduction}

In recent years the theory of fractional derivatives and integrals called Fractional Calculus has been steadily gaining importance for applications. Ordinary and partial differential equations of fractional order have been widely used for modeling various processes in physics, chemistry, aerodynamics of complex medium, polymer rheology, and control of dynamical systems (see, e.g., [1-3] and the references therein). Recently, many researchers paid attention to the existence of solutions of the initial value problems and boundary value problems for fractional differential equations, such as [411]. In [4], the existence and uniqueness of solution of the following initial value problem for fractional equation of Volterra type with the Riemann-Liouville derivative

$$
\begin{gathered}
D^{q} x(t)=f\left(t, x(t), \int_{0}^{t} k(t, s) x(s) d s\right), \\
t \in J_{0}=(0, T], \quad T>0, \\
\left.t^{1-q} x(t)\right|_{t=0}=r,
\end{gathered}
$$

was discussed by using the method of upper and lower solutions and its associated monotone iterative method. In [9], the existence and uniqueness of extremal solutions of the following system of nonlinear fractional differential equations

$$
\begin{aligned}
& D^{\alpha} u(t)=f(t, u(t), v(t)), \quad t \in(0, T], \\
& D^{\alpha} v(t)=g(t, v(t), u(t)), \quad t \in(0, T], \\
& \left.t^{1-\alpha} u(t)\right|_{t=0}=x_{0},\left.\quad t^{1-\alpha} v(t)\right|_{t=0}=y_{0}
\end{aligned}
$$

was discussed by using the same method, too.

Motivated by the above two papers, we consider the existence of solutions for a system of nonlinear fractional differential equations subject to initial conditions of the type

$$
\begin{gathered}
D^{\alpha} u(t)=f\left(t, u(t), \int_{0}^{t} k(t, s) v(s) d s\right), \quad t \in(0, T], \\
D^{\alpha} v(t)=g\left(t, v(t), \int_{0}^{t} k(t, s) u(s) d s\right), \quad t \in(0, T], \\
\left.t^{1-\alpha} u(t)\right|_{t=0}=x_{0},\left.\quad t^{1-\alpha} v(t)\right|_{t=0}=y_{0},
\end{gathered}
$$

where the parameter $0<\alpha \leq 1$ is the order of the fractional differential equations, and we assume that $0<T<\infty, f, g \in$ $C([0, T] \times \mathbb{R} \times \mathbb{R}, \mathbb{R}), x_{0}, y_{0} \in \mathbb{R}, x_{0} \leq y_{0} . D^{\alpha}$ is the standard Riemann-Liouville fractional derivative of order $0<\alpha \leq 1$ (see [1]). It is worthwhile to indicate that the nonlinear terms in the systems involve the unknown functions $u(t)$ and $v(t)$. 
The rest of this paper is organized as follows. In Section 2, some preliminary knowledge and the existence and uniqueness of solution for a linear problem for systems of differential equations are discussed and a differential inequality as a comparison principle is established. In Section 3, by using the monotone iterative technique and the method of upper and lower solutions, we prove the existence of extremal solutions of systems (3). Finally, an example is given to illustrate our results.

\section{Preliminaries}

In this section, we will state some necessary definitions and preliminary results which will be used in the next section to attain the existence of solutions for the nonlinear system (3).

First, consider the set $C_{1-\alpha}([0, T])=\{u \in C([0, T])$; $\left.t^{1-\alpha} u \in C([0, T])\right\}$. For $u \in C_{1-\alpha}([0, T])$ we define two weighted norms:

$$
\|u\|^{*}=\max _{t \in[0, T]} t^{1-\alpha}|u(t)|, \quad\|u\|_{*}=\max _{t \in[0, T]} t^{1-\alpha} e^{-\lambda t}|u(t)|,
$$

with a fixed positive constant $\lambda$.

Now we enunciate the following existence and uniqueness results for the initial value problem (IVP) of the linear fractional differential equations. For the following IVP of fractional differential equation

$$
D^{\alpha} x=f(t, x), \quad x\left(t_{0}\right)=x^{0}=\left.x(t)\left(t-t_{0}\right)^{1-\alpha}\right|_{t=t_{0}},
$$

where $f \in C([0, T] \times \mathbb{R})$, it is equivalent to the following Volterra integral equation:

$$
x(t)=x^{0}(t)+\frac{1}{\Gamma(\alpha)} \int_{t_{0}}^{t}(t-s)^{\alpha-1} f(s, x(s)) d s .
$$

Lemma 1 (see $[12])$. Let $m \in C_{1-\alpha}([0, T], \mathbb{R})$ be locally Hölder continuous function such that, for any $t_{1} \in(0, T]$, one has

$$
m\left(t_{1}\right)=0, \quad m(t) \leq 0 \quad \text { for } t_{0} \leq t \leq t_{1} .
$$

Then it follows that

$$
D^{\alpha} m\left(t_{1}\right) \geq 0 .
$$

Lemma 2. Let $\alpha \in(0,1), M \in \mathbb{R}, N \in \mathbb{R}, k(t, s) \in$ $C\left((0, T] \times(0, T], \mathbb{R}^{+}\right)$, and $|k(t, s)| \leq K, \sigma \in C_{1-\alpha}((0, T], \mathbb{R})$. In addition, one assumes that

$\left(H_{1}\right)$

$$
\frac{T^{\alpha} \Gamma(\alpha)}{\Gamma(2 \alpha)}\left(|M|+\frac{|N| k T}{2 \alpha}\right)<1, \quad \text { if } 0<\alpha \leq \frac{1}{2} .
$$

Then the IVP

$$
\begin{gathered}
D^{\alpha} x(t)=\sigma(t)-M x(t)-N \int_{0}^{t} k(t, s) x(s) d s, \quad t \in(0, T], \\
\left.t^{1-\alpha} x(t)\right|_{t=0}=r
\end{gathered}
$$

has unique solution.
Proof. In the case when $1 / 2<\alpha \leq 1$, we use the norm $\|\cdot\|_{*}$ with positive number $\lambda$ satisfying $\sqrt{\lambda}>\rho_{1} \equiv$ $(\Gamma(2 \alpha-1) / \sqrt{2 \Gamma(2(2 \alpha-1))})((|M|+|N| K T) / \alpha \Gamma(\alpha)) \sqrt{T^{2 \alpha-1}}$. The remainder part of the case and the case of $0<\alpha \leq 1 / 2$ are similar to that of Theorem 1 in paper [4], so we omit the details.

Lemma 3. Suppose that condition $\left(H_{1}\right)$ holds. Let $0<\alpha \leq 1$, $M, N \in \mathbb{R}$, and $\sigma_{1}, \sigma_{2} \in C_{1-\alpha}([0, T])$; then the IVP

$$
\begin{aligned}
D^{\alpha} u(t)= & \sigma_{1}(t)-M u(t)-N \int_{0}^{t} k(t, s) v(s) d s, \quad t \in(0, T], \\
D^{\alpha} v(t)= & \sigma_{2}(t)-M v(t)-N \int_{0}^{t} k(t, s) u(s) d s, \quad t \in(0, T], \\
& \left.t^{1-\alpha} u(t)\right|_{t=0}=x_{0},\left.\quad t^{1-\alpha} v(t)\right|_{t=0}=y_{0}
\end{aligned}
$$

has unique system of solutions in $C_{1-\alpha}([0, T]) \times C_{1-\alpha}([0, T])$.

Proof. The proof follows from the fact that the pair $(u, v)$ is a solution of problem (11) if and only if $u(t)$ and $v(t)$ have the form

$$
u(t)=\frac{p(t)+q(t)}{2}, \quad v(t)=\frac{p(t)-q(t)}{2}, \quad t \in[0, T],
$$

where $p$ and $q$ solve the problems

$$
\begin{gathered}
D^{\alpha} p(t)=\left(\sigma_{1}+\sigma_{2}\right)(t)-M p(t)-N \int_{0}^{t} k(t, s) p(s) d s, \\
t \in(0, T], \\
\left.t^{1-\alpha} p(t)\right|_{t=0}=x_{0}+y_{0}, \\
D^{\alpha} q(t)=\left(\sigma_{1}-\sigma_{2}\right)(t)-M q(t)-N \int_{0}^{t} k(t, s) q(s) d s, \\
\left.t^{1-\alpha} q(t)\right|_{t=0}=x_{0}-y_{0} .
\end{gathered}
$$

By Lemma 2, we know that both problems (13) and (14) have unique solution in $C_{1-\alpha}([0, T])$. Consequently, $u$ and $v$ are uniquely determined, too. This completes the proof of the lemma.

Lemma 4. Let $0<\alpha \leq 1, M>0, N \in \mathbb{R}, w \in C_{1-\alpha}([0, T])$ and let $w(t)$ be locally Hölder continuous function such that

$$
\begin{gathered}
D^{\alpha} w(t)+M w(t)+N \int_{0}^{t} k(t, s) w(s) d s \geq 0, \quad t \in(0, T] \\
\left.t^{1-\alpha} w(t)\right|_{t=0}=w_{0} \geq 0 .
\end{gathered}
$$

Then $w(t) \geq 0$ for all $t \in(0, T]$. 
Proof. Assume that the assertion is not true. Then from $\left.t^{1-\alpha} w(t)\right|_{t=0}=w_{0} \geq 0$, there exist points $t_{0}, t_{0}^{\prime} \in(0, T]$ such that $w\left(t_{0}\right)=0, w\left(t_{0}^{\prime}\right)<0$, and $w(t) \geq 0$, for $t \in\left(0, t_{0}\right]$, and $w(t)<0$, for $t \in\left(t_{0}, t_{0}^{\prime}\right]$. Assume that $t_{1}$ is the first minimal point of $w(t)$ on $\left[t_{0}, t_{0}^{\prime}\right]$. We divide the reminder of the proof into two separate cases.

Case 1. Let $M>0, N<0$. It follows from Lemma 1 that

$$
D^{\alpha}\left(-w\left(t_{0}\right)\right) \geq 0 \text {. }
$$

Hence, we have

$$
D^{\alpha} w\left(t_{0}\right) \leq 0
$$

However,

$$
D^{\alpha} w\left(t_{0}\right)+M w\left(t_{0}\right)+N \int_{0}^{t_{0}} k\left(t_{0}, s\right) w(s) d s \geq 0 .
$$

So, we have

$$
D^{\alpha} w\left(t_{0}\right) \geq-M w\left(t_{0}\right)-N \int_{0}^{t_{0}} k\left(t_{0}, s\right) w(s) d s>0,
$$

which is a contradiction. So the assertion holds in this case.

Case 2. Let $M>0, N>0$. From the condition $D^{\alpha} w(t)+$ $M w(t)+N \int_{0}^{t} k(t, s) w(s) d s \geq 0$, we have

$$
D^{\alpha} w(t) \geq 0
$$

for all $t \in\left(t_{0}, t_{0}^{\prime}\right]$. Hence

$$
\int_{t_{0}}^{t} D^{\alpha} w(s) d s \geq 0, \quad \forall t \in\left(t_{0}, t_{0}^{\prime}\right] .
$$

That is,

$$
I^{1-\alpha} w(t)-I^{1-\alpha} w\left(t_{0}\right) \geq 0, \quad \forall t \in\left(t_{0}, t_{0}^{\prime}\right] .
$$

On the other hand, for $t \in\left(t_{0}, t_{0}^{\prime}\right]$,

$$
\begin{aligned}
I^{1-\alpha} w(t)-I^{1-\alpha} w\left(t_{0}\right) \\
=\frac{1}{\Gamma(1-\alpha)} \int_{0}^{t}(t-s)^{-\alpha} w(s) d s \\
\quad-\frac{1}{\Gamma(1-\alpha)} \int_{0}^{t_{0}}\left(t_{0}-s\right)^{-\alpha} w(s) d s \\
=\frac{1}{\Gamma(1-\alpha)} \int_{0}^{t_{0}}\left((t-s)^{-\alpha}-\left(t_{0}-s\right)^{-\alpha}\right) w(s) d s \\
\quad+\frac{1}{\Gamma(1-\alpha)} \int_{t_{0}}^{t}(t-s)^{-\alpha} w(s) d s \\
<0,
\end{aligned}
$$

which contradicts with (22), so the assertion holds.
Lemma 5. Let $0<\alpha \leq 1, M \geq 0, N>0$, and $k(t, s) \in$ $C\left((0, T] \times(0, T], \mathbb{R}^{+}\right), u, v \in C_{1-\alpha}([0, T])$. Moreover $u(t), v(t)$ are locally Hölder continuous functions such that

$$
\begin{gathered}
D^{\alpha} u(t) \geq-M u(t)+N \int_{0}^{t} k(t, s) v(s) d s, \quad t \in(0, T], \\
D^{\alpha} v(t) \geq-M v(t)+N \int_{0}^{t} k(t, s) u(s) d s, \quad t \in(0, T], \\
\left.t^{1-\alpha} u(t)\right|_{t=0}=x_{0} \geq 0, \\
\left.t^{1-\alpha} v(t)\right|_{t=0}=y_{0} \geq 0 .
\end{gathered}
$$

Then for all $t \in(0, T]$, we have $u(t) \geq 0, v(t) \geq 0$.

Proof. Let $p(t)=u(t)+v(t), \forall t \in(0, T]$. By (24) we have

$$
\begin{gathered}
D^{\alpha} p(t) \geq-M p(t)+N \int_{0}^{t} k(t, s) p(s) d s, \quad t \in(0, T] \\
\left.t^{1-\alpha} p(t)\right|_{t=0} \geq 0 .
\end{gathered}
$$

Hence

$$
p(t) \geq 0
$$

That is,

$$
u(t) \geq-v(t) .
$$

In fact, by (24) and (27), we have that

$$
\begin{gathered}
D^{\alpha} u(t) \geq-M u(t)-N \int_{0}^{t} k(t, s) u(s) d s, \quad t \in(0, T], \\
\left.t^{1-\alpha} u(t)\right|_{t=0} \geq 0, \\
D^{\alpha} v(t) \geq-M v(t)-N \int_{0}^{t} k(t, s) v(s) d s, \quad t \in(0, T], \\
\left.t^{1-\alpha} v(t)\right|_{t=0} \geq 0 .
\end{gathered}
$$

From Lemma 4, we obtain $u(t) \geq 0, v(t) \geq 0, \forall t \in(0, T]$. This completes the proof of the lemma.

\section{Main Results}

In this section, we prove the existence of extremal solutions of nonlinear system (3). We list the following assumptions for convenience. 
$\left(H_{2}\right)$ The function $k(t, s) \in C\left((0, T] \times(0, T], \mathbb{R}^{+}\right)$. There exist $u_{0}, v_{0} \in C_{1-\alpha}([0, T])$, which are locally Hölder continuous functions, and $u_{0} \leq v_{0}$, such that

$$
\begin{gathered}
D^{\alpha} u_{0}(t) \leq f\left(t, u_{0}(t), \int_{0}^{t} k(t, s) v_{0}(s) d s\right), \quad t \in(0, T], \\
\left.t^{1-\alpha} u_{0}(t)\right|_{t=0} \leq x_{0}, \\
D^{\alpha} v_{0}(t) \geq g\left(t, v_{0}(t), \int_{0}^{t} k(t, s) u_{0}(s) d s\right), \quad t \in(0, T], \\
\left.t^{1-\alpha} v_{0}(t)\right|_{t=0} \geq y_{0} .
\end{gathered}
$$

$\left(H_{3}\right)$ There exist $M \geq 0, N \geq 0$, such that

$$
\begin{gathered}
f\left(t, u(t), \int_{0}^{t} k(t, s) v(s) d s\right)-f\left(t, \bar{u}(t), \int_{0}^{t} k(t, s) \bar{v}(s) d s\right) \\
\geq-M(u(t)-\bar{u}(t))-N \int_{0}^{t} k(t, s)(v(s)-\bar{v}(s)) d s, \\
g\left(t, v(t), \int_{0}^{t} k(t, s) u(s) d s\right)-g\left(t, \bar{v}(t), \int_{0}^{t} k(t, s) \bar{u}(s) d s\right) \\
\quad \geq-M(v(t)-\bar{v}(t))-N \int_{0}^{t} k(t, s)(u(s)-\bar{u}(s)) d s,
\end{gathered}
$$

where $u_{0}(t) \leq \bar{u}(t) \leq u(t) \leq v_{0}(t), u_{0}(t) \leq$ $v(t) \leq \bar{v}(t) \leq v_{0}(t)$, and $g\left(t, v(t), \int_{0}^{t} k(t, s) u(s) d s\right)$ - $f\left(t, u(t), \int_{0}^{t} k(t, s) v(s) d s\right) \geq M(u(t)-v(t))+$ $N \int_{0}^{t} k(t, s)(v(s)-u(s)) d s$, with $u_{0}(t) \leq u(t) \leq v(t) \leq$ $v_{0}(t)$.

Theorem 6. Suppose that conditions $\left(H_{1}\right)-\left(H_{3}\right)$ hold. Then, there exists an $\left(u^{*}, v^{*}\right) \in\left[u_{0}, v_{0}\right] \times\left[u_{0}, v_{0}\right]$ which is an extremal solution of the nonlinear problem (3). Moreover, there exist monotone iterative sequences $\left\{u_{n}\right\},\left\{v_{n}\right\} \subset\left[u_{0}, v_{0}\right]$, such that $\left\{u_{n}\right\} \rightarrow u^{*},\left\{v_{n}\right\} \rightarrow v^{*}(n \rightarrow \infty)$ uniformly on $t \in(0, T]$, and

$$
u_{0} \leq u_{1} \leq \cdots \leq u_{n} \leq u^{*} \leq v^{*} \leq \cdots \leq v_{n} \leq \cdots \leq v_{1} \leq v_{0} .
$$

Proof. First, for any $u_{n-1}, v_{n-1} \in C_{1-\alpha}([0, T]), n \geq 1$, we consider the IVP of the linear system

$$
\begin{aligned}
D^{\alpha} u_{n}(t)= & f\left(t, u_{n-1}(t), \int_{0}^{t} k(t, s) v_{n-1}(s) d s\right) \\
& +M u_{n-1}(t)+N \int_{0}^{t} k(t, s) v_{n-1}(s) d s \\
& -M u_{n}(t)-N \int_{0}^{t} k(t, s) v_{n}(s) d s, \quad t \in(0, T],
\end{aligned}
$$

$$
\begin{aligned}
& D^{\alpha} v_{n}(t)= g\left(t, v_{n-1}(t), \int_{0}^{t} k(t, s) u_{n-1}(s) d s\right) \\
&+M v_{n-1}(t)+N \int_{0}^{t} k(t, s) u_{n-1}(s) d s \\
&-M v_{n}(t)-N \int_{0}^{t} k(t, s) u_{n}(s) d s, \quad t \in(0, T], \\
&\left.t^{1-\alpha} u_{n}(t)\right|_{t=0}=x_{0},\left.\quad t^{1-\alpha} v_{n}(t)\right|_{t=0}=y_{0} .
\end{aligned}
$$

From Lemma 3, we know that (32) has unique system of solutions in $C_{1-\alpha}([0, T]) \times C_{1-\alpha}([0, T])$.

Next, we show that $\left\{u_{n}(t)\right\},\left\{v_{n}(t)\right\}$ satisfy the property

$$
u_{n-1} \leq u_{n} \leq v_{n} \leq v_{n-1}, \quad n=1,2, \ldots
$$

Let $p(t)=u_{1}(t)-u_{0}(t), q(t)=v_{0}(t)-v_{1}(t)$. From (32) and $\left(\mathrm{H}_{2}\right)$, we have that

$$
\begin{aligned}
D^{\alpha} p(t)= & D^{\alpha} u_{1}(t)-D^{\alpha} u_{0}(t) \\
\geq & f\left(t, u_{0}(t), \int_{0}^{t} k(t, s) v_{0}(s) d s\right)+M u_{0}(t) \\
& +N \int_{0}^{t} k(t, s) v_{0}(s) d s-M u_{1}(t) \\
& -N \int_{0}^{t} k(t, s) v_{1}(s) d s \\
& -f\left(t, u_{0}(t), \int_{0}^{t} k(t, s) v_{0}(s) d s\right) \\
= & -M p(t)+N \int_{0}^{t} k(t, s) q(s) d s, \\
D^{\alpha} q(t)= & D^{\alpha} v_{0}(t)-D^{\alpha} v_{1}(t) \\
\geq & g\left(t, v_{0}(t), \int_{0}^{t} k(t, s) u_{0}(s) d s\right) \\
& -g\left(t, v_{0}(t), \int_{0}^{t} k(t, s) u_{0}(s) d s\right)-M v_{0}(t) \\
& -N \int_{0}^{t} k(t, s) v_{0}(s) d s+M v_{1}(t) \\
& +N \int_{0}^{t} k(t, s) u_{1}(s) d s \\
= & -M q(t)+N \int_{0}^{t} k(t, s) p(s) d s, \\
\left.t^{1-\alpha} p(t)\right|_{t=0} \geq & x_{0}-x_{0}=0, \\
& \left.t^{1-\alpha} q(t)\right|_{t=0} \geq y_{0}-y_{0}=0 .
\end{aligned}
$$

Thus, by Lemma 5, we have that $p(t) \geq 0, q(t) \geq 0, \forall t \in$ $(0, T]$. 
Let $w(t)=v_{1}(t)-u_{1}(t)$. By condition (32) and $\left(H_{3}\right)$, we obtain

$$
\begin{aligned}
D^{\alpha} w(t)= & D^{\alpha} v_{1}(t)-D^{\alpha} u_{1}(t) \\
= & g\left(t, v_{0}(t), \int_{0}^{t} k(t, s) u_{0}(s) d s\right)+M v_{0}(t) \\
& +N \int_{0}^{t} k(t, s) u_{0}(s) d s-M v_{1}(t) \\
& -N \int_{0}^{t} k(t, s) u_{1}(s) d s \\
& -f\left(t, u_{0}(t), \int_{0}^{t} k(t, s) v_{0}(s) d s\right)-M u_{0}(t) \\
& -N \int_{0}^{t} k(t, s) v_{0}(s) d s+M u_{1}(t) \\
& +N \int_{0}^{t} k(t, s) v_{1}(s) d s \\
\geq & -M w(t)+N \int_{0}^{t} k(t, s) w(s) d s, \\
& \left.t^{1-\alpha} w(t)\right|_{t=0}=y_{0}-x_{0} \geq 0 .
\end{aligned}
$$

By Lemma 4 , we obtain $w(t) \geq 0, \forall t \in(0, T]$. Hence, we have the relation $u_{0} \leq u_{1} \leq v_{1} \leq v_{0}$.

Now, we assume that $u_{k-1} \leq u_{k} \leq v_{k} \leq v_{k-1}$, for some $k \geq 1$, and we prove that (33) is true for $k+1$, too. Let $p(t)=$ $u_{k+1}(t)-u_{k}(t), q(t)=v_{k}(t)-v_{k+1}(t), w(t)=v_{k+1}(t)-u_{k+1}(t)$. By (32) and $\left(H_{3}\right)$, we have that

$$
\begin{aligned}
D^{\alpha} p(t)= & D^{\alpha} u_{k+1}(t)-D^{\alpha} u_{k}(t) \\
= & f\left(t, u_{k}(t), \int_{0}^{t} k(t, s) v_{k}(s) d s\right)+M u_{k}(t) \\
& +N \int_{0}^{t} k(t, s) v_{k}(s) d s-M u_{k+1}(t) \\
& -N \int_{0}^{t} k(t, s) v_{k+1}(s) d s \\
& -f\left(t, u_{k-1}(t), \int_{0}^{t} k(t, s) v_{k-1}(s) d s\right) \\
& -M u_{k-1}(t)-N \int_{0}^{t} k(t, s) v_{k-1}(s) d s+M u_{k}(t) \\
& +N \int_{0}^{t} k(t, s) v_{k}(s) d s \\
\geq & -M p(t)+N \int_{0}^{t} k(t, s) q(s) d s, \quad t \in(0, T],
\end{aligned}
$$

$$
\begin{aligned}
& D^{\alpha} q(t)=D^{\alpha} v_{k}(t)-D^{\alpha} v_{k+1}(t) \\
& =g\left(t, v_{k-1}(t), \int_{0}^{t} k(t, s) u_{k-1}(s) d s\right)+M v_{k-1}(t) \\
& +N \int_{0}^{t} k(t, s) u_{k-1}(s) d s-M v_{k}(t) \\
& -N \int_{0}^{t} k(t, s) u_{k}(s) d s \\
& -g\left(t, v_{k}(t), \int_{0}^{t} k(t, s) u_{k}(s) d s\right)-M v_{k}(t) \\
& -N \int_{0}^{t} k(t, s) u_{k}(s) d s+M v_{k+1}(t) \\
& +N \int_{0}^{t} k(t, s) u_{k+1}(s) d s \\
& \geq-M q(t)+N \int_{0}^{t} k(t, s) p(s) d s, \quad t \in(0, T], \\
& \left.t^{1-\alpha} p(t)\right|_{t=0}=0,\left.\quad t^{1-\alpha} q(t)\right|_{t=0}=0, \\
& D^{\alpha} w(t) \geq-M w(t)+N \int_{0}^{t} k(t, s) w(s) d s, \quad t \in(0, T], \\
& \left.t^{1-\alpha} w(t)\right|_{t=0} \geq 0 \text {. }
\end{aligned}
$$

By Lemmas 4 and 5, we have that $u_{k} \leq u_{k+1} \leq v_{k+1} \leq v_{k}$.

From the above, by induction, it is easy to prove that

$$
u_{0} \leq u_{1} \leq \cdots \leq u_{n} \leq \cdots \leq v_{n} \leq \cdots \leq v_{1} \leq v_{0} .
$$

We see that $\left\{u_{n}\right\}$ is monotone nondecreasing and is bounded from above and $\left\{v_{n}\right\}$ is monotone nonincreasing and is bounded from below; hence,

$$
\lim _{n \rightarrow \infty} u_{n}(t)=u^{*}, \quad \lim _{n \rightarrow \infty} v_{n}(t)=v^{*},
$$

uniformly on compact subsets of $(0, T]$, and the limit functions $u^{*}, v^{*}$ satisfy (3). Moreover, $u^{*}, v^{*} \in\left[u_{0}, v_{0}\right]$. Taking the limits in (32), we know that $\left(u^{*}, v^{*}\right)$ is a system of solutions of (3) in $\left[u_{0}, v_{0}\right] \times\left[u_{0}, v_{0}\right]$. Moreover, (31) is true.

Finally, we prove that (3) has an extremal solution. Assume that $(u, v) \in\left[u_{0}, v_{0}\right] \times\left[u_{0}, v_{0}\right]$ is any solutions of $(3)$. That is,

$$
\begin{gathered}
D^{\alpha} u(t)=f\left(t, u(t), \int_{0}^{t} k(t, s) v(s) d s\right), \quad t \in(0, T], \\
D^{\alpha} v(t)=g\left(t, v(t), \int_{0}^{t} k(t, s) u(s) d s\right), \quad t \in(0, T], \\
\left.t^{1-\alpha} u(t)\right|_{t=0}=x_{0},\left.\quad t^{1-\alpha} v(t)\right|_{t=0}=y_{0} .
\end{gathered}
$$

By (32), (39), $\left(\mathrm{H}_{3}\right)$, and Lemma 5 , it is easy to prove that

$$
u_{n} \leq u, v \leq v_{n}, \quad n=1,2, \ldots
$$


By taking the limits in (40) as $n \rightarrow \infty$, we have that $u^{*} \leq$ $u, v \leq v^{*}$. That is, $\left(u^{*}, v^{*}\right)$ is an extremal solution of (3) in $\left[u_{0}, v_{0}\right] \times\left[u_{0}, v_{0}\right]$. This completes the proof.

\section{An Example}

Example 1. Consider the following problem:

$$
\begin{gathered}
D^{\alpha} u(t)=M t^{2}[t-u(t)]-N \int_{0}^{t}(t+s) v(s) d s, \\
D^{\alpha} v(t)=M t^{2}[t-v(t)]-N \int_{0}^{t}(t+s) u(s) d s, \\
\left.t^{1-\alpha} u(t)\right|_{t=0}=0,\left.\quad t^{1-\alpha} v(t)\right|_{t=0}=0,
\end{gathered}
$$

where $t \in[0,1], D^{\alpha}$ is the standard Riemann-Liouville fractional derivative of order $0<\alpha \leq 1$, and $M, N$ are constants satisfying $M>(5 / 6) N$. In view of Lemma 3 , the nonlinear system (41) has unique solution if $1 / 2<\alpha \leq 1$, and in case of $0<\alpha \leq 1 / 2$, an additional assumption $(\Gamma(\alpha) / \Gamma(2 \alpha))(|M|+|N| / \alpha)<1$ is added so that $\left(H_{1}\right)$ holds. Note that in a special case, let $M=\alpha^{K}, N=\alpha^{K+1}$, where $K>$ 0 is a sufficiently large real number; $\left(H_{1}\right)$ holds automatically. Obviously,

$$
\begin{aligned}
& f\left(t, u(t), \int_{0}^{t} k(t, s) v(s) d s\right) \\
& =M t^{2}[t-u(t)]-N \int_{0}^{t}(t+s) v(s) d s, \\
& g\left(t, v(t), \int_{0}^{t} k(t, s) u(s) d s\right) \\
& =M t^{2}[t-v(t)]-N \int_{0}^{t}(t+s) u(s) d s .
\end{aligned}
$$

Take $u_{0}(t)=0, v_{0}(t)=t$; then

$$
\begin{aligned}
& D^{\alpha} u_{0}(t)=0 \leq\left(M-\frac{5}{6} N\right) t^{3} \\
&=f\left(t, u_{0}(t), \int_{0}^{t} k(t, s) v_{0}(s) d s\right), \\
& D^{\alpha} v_{0}(t)=\frac{t^{1-\alpha}}{\Gamma(2-\alpha)} \geq 0 \\
&=g\left(t, v_{0}(t), \int_{0}^{t} k(t, s) u_{0}(s) d s\right), \\
&\left.t^{1-\alpha} u(t)\right|_{t=0}=\left.t^{1-\alpha} v(t)\right|_{t=0}=0 .
\end{aligned}
$$

So condition $\left(\mathrm{H}_{2}\right)$ of Theorem 6 holds. Moreover, it is easy to verify that condition $\left(\mathrm{H}_{3}\right)$ holds; thus, all conditions of Theorem 6 are satisfied. Consequently, the nonlinear system (41) has an extremal solution $\left(u^{*}, v^{*}\right) \in[0, t] \times[0, t]$.

\section{Conflict of Interests}

The authors declare that there is no conflict of interests regarding the publication of this paper.

\section{Acknowledgments}

The authors thank the referee for his helpful suggestions which improved some of their results. This research was partially supported by the NSF of China (Grants 11271225, 11171178), Science and Technology Project of High Schools of Shandong Province (Grant J12LI52) and Program for Scientific research innovation team in colleges and universities of Shandong Province.

\section{References}

[1] A. A. Kilbas, H. M. Srivastava, and J. J. Trujillo, Theory and Applications of Fractional Differential Equations, vol. 204 of North-Holland Mathematics Studies, Elsevier Science, Amsterdam, The Netherlands, 2006.

[2] V. Lakshmikantham, S. Leela, and J. Vasundhara, Theory of Fractional Dynamic Systems, vol. 11 of Systems of Nonlinear Fractional Differential Equations, Cambridge Academic Publishers, Cambridge, UK, 2009.

[3] I. Podlubny, Fractional Differential Equations, vol. 198 of Mathematics in Science and Engineering, Academic Press, San Diego, Calif, USA, 1999.

[4] T. Jankowski, "Fractional equations of Volterra type involving a Riemann-Liouville derivative," Applied Mathematics Letters, vol. 26, no. 3, pp. 344-350, 2013.

[5] X. Su, "Boundary value problem for a coupled system of nonlinear fractional differential equations," Applied Mathematics Letters, vol. 22, no. 1, pp. 64-69, 2009.

[6] F. A. McRae, "Monotone iterative technique and existence results for fractional differential equations," Nonlinear Analysis: Theory, Methods \& Applications, vol. 71, no. 12, pp. 6093-6096, 2009.

[7] J. D. Ramírez and A. S. Vatsala, "Monotone iterative technique for fractional differential equations with periodic boundary conditions," Opuscula Mathematica, vol. 29, no. 3, pp. 289-304, 2009.

[8] G. Wang, "Monotone iterative technique for boundary value problems of a nonlinear fractional differential equation with deviating arguments," Journal of Computational and Applied Mathematics, vol. 236, no. 9, pp. 2425-2430, 2012.

[9] G. Wang, R. P. Agarwal, and A. Cabada, "Existence results and the monotone iterative technique for systems of nonlinear fractional differential equations," Applied Mathematics Letters, vol. 25, no. 6, pp. 1019-1024, 2012.

[10] Z. Wei, Q. Li, and J. Che, "Initial value problems for fractional differential equations involving Riemann-Liouville sequential fractional derivative," Journal of Mathematical Analysis and Applications, vol. 367, no. 1, pp. 260-272, 2010.

[11] S. Zhang, "Monotone iterative method for initial value problem involving Riemann-Liouville fractional derivatives," Nonlinear Analysis: Theory, Methods \& Applications, vol. 71, no. 5-6, pp. 2087-2093, 2009.

[12] V. Lakshmikantham and A. S. Vatsala, "Theory of fractional differential inequalities and applications," Communications in Applied Analysis, vol. 11, no. 3-4, pp. 395-402, 2007. 


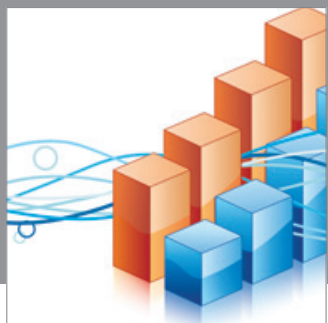

Advances in

Operations Research

mansans

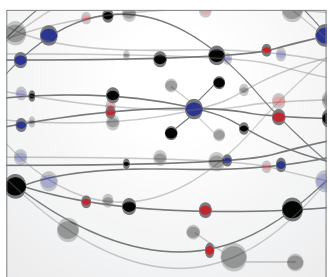

The Scientific World Journal
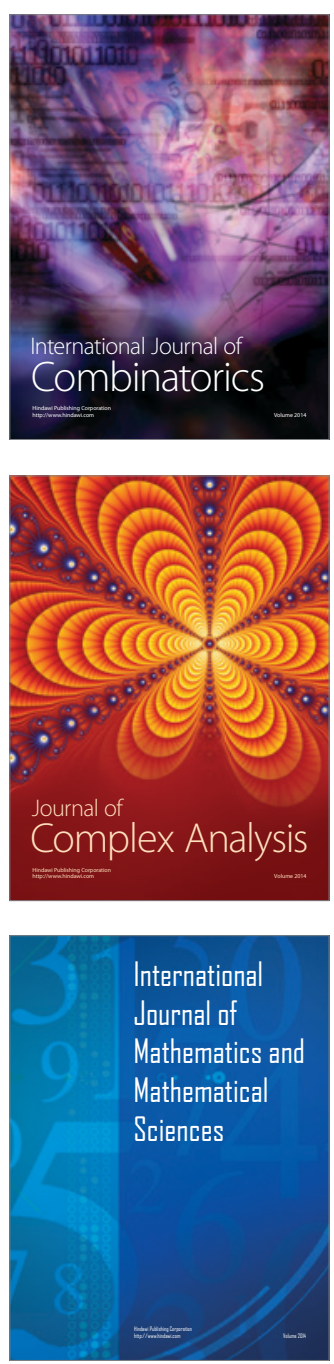
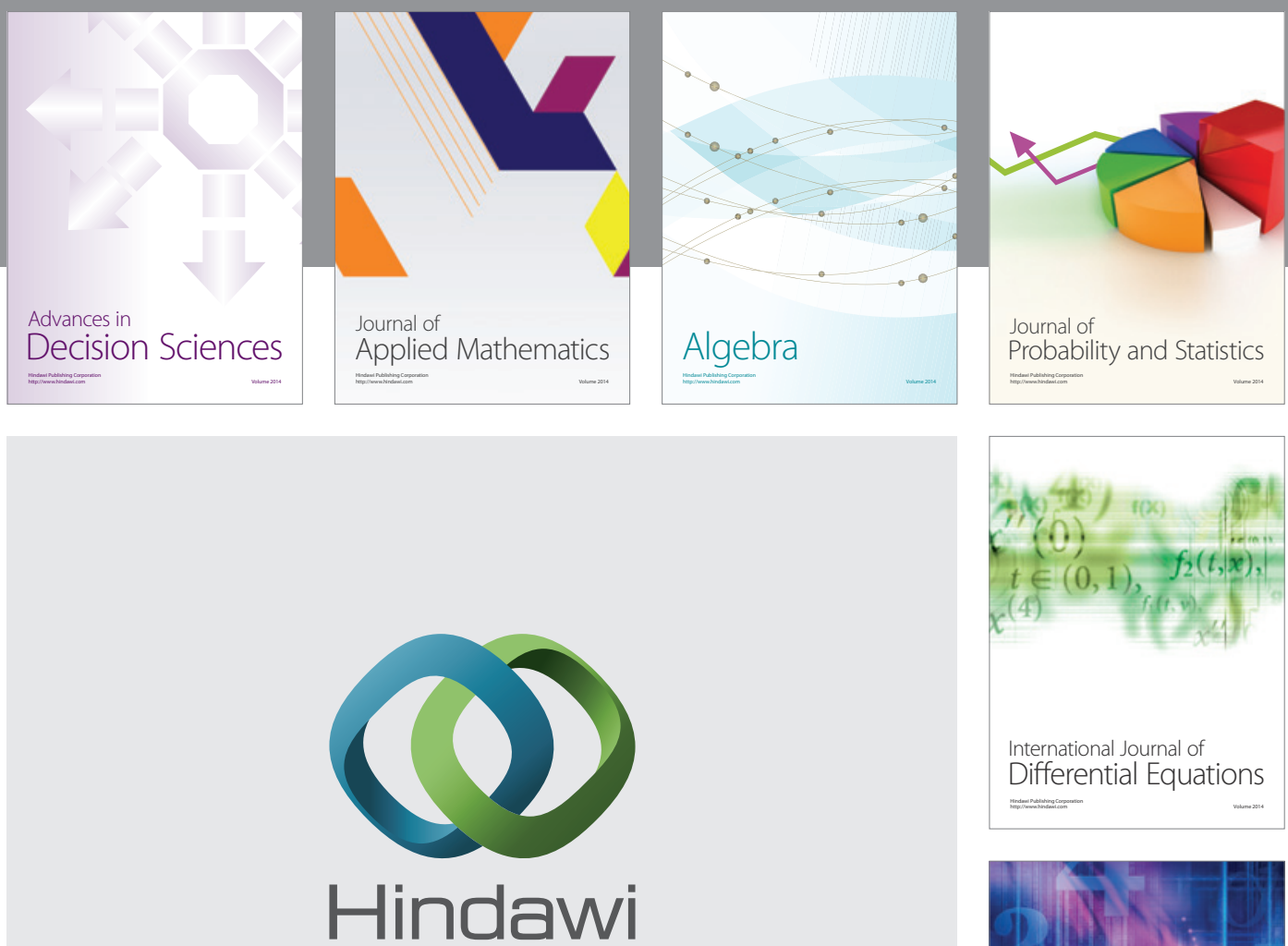

Submit your manuscripts at http://www.hindawi.com
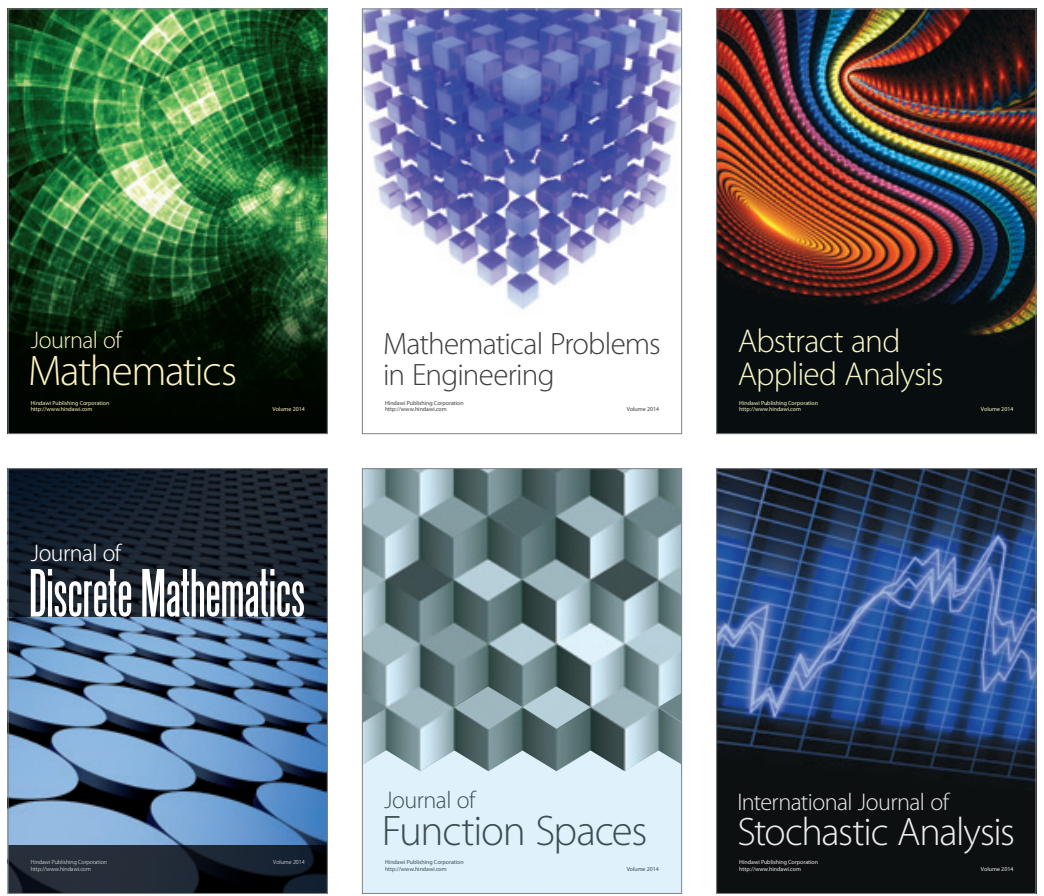

Journal of

Function Spaces

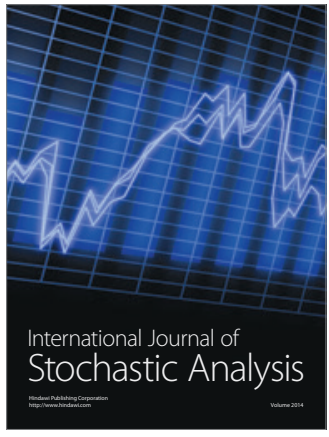

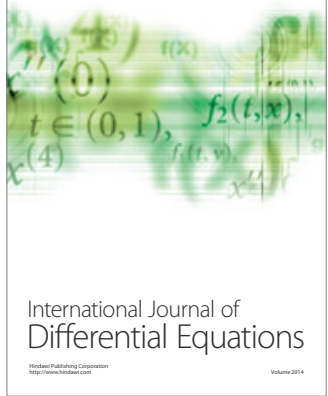
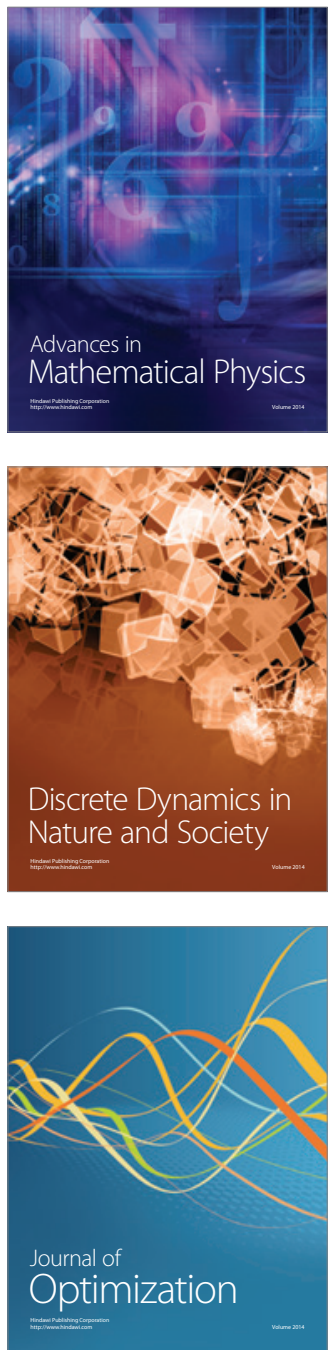